\title{
Patología placentaria de las toxemias del embarazo
}

\author{
Doctor Oscar Henao-Cabal
}

Auxiliar de Cátedra de Obstetricia. Facultad de Medicina Universidad del Valle.

\section{Doctor Saulo Muñoz-Delgado}

Auxiliar de Cátedra de Obstetricia. Facultad de Medicina Universidad del Valle.

Los investigadores buscan en la placenta la explicación al problema de la etiología de las toxemias. Es lógico que se piense así, ya que la placenta viene a ser el eslabón en una serie de hechos clínicos, sin cuya presencia no se lograrían encadenar.

Estudios de Dieckmann y Page afirman que "hay que darle más importancia al tejido placentario en relación con la etiología de la preeclampsia-eclampsia, debido a que hay suficientes evidencias que lo indican de esta manera".

A medida que se han profundizado los estudios de patología placentaria y con comprobaciones bioquímicas e histoquímicas, se ha ido encontrado la explicación a innumerables hechos hasta ahora oscuros. Como bien lo afirma Bartholomew; uno de los investigadores en la patología de la placenta, los cambios hallados en ésta, son otra pieza en el rompecabezas del mecanismo del desarrollo de la preeclampsia-eclapsia.

Los más recientes estudios, han dirigido su atención hacia los cambios patológicos placentarios, como factor etiológico del papel que desempeña la placenta.

Una de las teorías más posibles en la producción de la eclampsia; es la placenta como fuente o factor en la producción cie sustancias, ya sean tóxicas, alérgicas, vasoconstrictoras u hormonales.

Burstein y asociados atribuyen a la placenta las manifestaciones clínicas que pueden producirse de la siguiente manera:

1ạ Por efectos tóxicos o alérgicos de productos de degeneración placentaria. 
2a Elaboración de una sustancia presora como el resultado de la isquemia uterina.

3a Aumento en la elaboración de sustancias tromboplásticas como consecuencia de la isquemia placentaria.

4ạ Alteración en el metabolismo hormonal.

Bartholomew y colaboradores se muestran inclinados hacia el papel etiológico que desempeñan las lesiones placentarias en la producción de la preeclampsia, eclampsia, y abruptio placentae. Creen que cuando se adelanten mejores estudios bioquímicos de las placentas de las toxémicas, será posible identificar la naturaleza y los efectos de otros productos de degradación de la necrosis placentaria.

Otra de las causas que han contribuido en la disparidad de conceptos sobre la patología placentaria en toxemias, ha sido la falta de descripción exacta de las múltiples lesiones e infartos y el atribuirle a la toxemia todas las lesiones sin hacer una identificación exacta de cada una.

Creemos conveniente, por lo tanto, especificar claramente los diferentes tipos de infartos para considerar su presencia en los casos de toxemia.

Después de una revisión bibliográfica hemos creido que la clasificación propuesta por Bartholomew, aun cuando es difícil, llena los objetivos perseguidos, ya que identifica las lesiones consecutivas a la toxemia y las diferencia de las frecuentemente halladas en los casos normales. Page opina que aun cuando la incidencia de degeneración es indudablemente mayor en la placenta toxémica que en la sana, ello no ocurre con la regularidad patognomónica proclamada por Bartholomew.

Nosotros no queremos especular sobre si estas lesiones placentarias sean ciertamente la causa misma de la toxemia, según las aseveraciones de Bartholomew, o si son simplemente el efecto de la toxemia sobre los vasos placentarios. El hecho demostrado desde Hertig es el de que hay cambios microscópicos en los pequeños vasos de la decidua materna y que aun cuando en cualquier placenta, de embarazada normal o toxémica, son demostrables numerosas y variadas alteraciones patológicas, solo raramente han sido apreciadas lesiones vasculares en placentas normales y en cambio en los $3 / 4$ de las placentas toxémicas, aun de pre-eclampsia leve, son demostrables cambios ateromatosos agudos que a veces llegan a la oclusión vascular completa. En todo caso, hemos tratado de, sin llegar a la intimidad de este problema, mostrar la frecuencia con que en nuestro medio se 


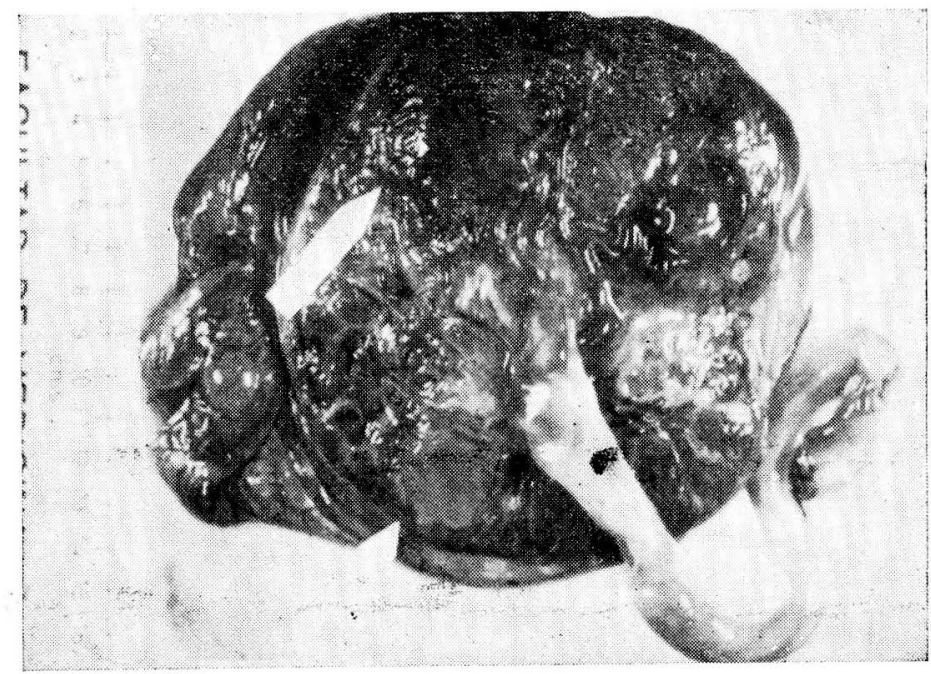

PLACENTA.-Aspecto macroscópico. Depósitos de fibrina subcoriónica.

presentan estas lesiones y aportar un número de estudio de placentas.

Han sido descritas innumerables lesiones placentarias en toxemia como nódulos, infartos, calcificaciones, etc., presentes en los $3 / 4$ de las placentas toxémicas. Por lo tanto, es importante diferenciar entre los verdaderos infartos y otras lesiones de la placenta. Desde los estudios de Young y con los aportes posteriores de Bartholomew se ha comprobado plenamente el tipo agudo de infarto placentario en las toxemias del embarazo.

Ha designado estos tipos de lesiones con las letras A, B, C, D, E, F, G y H, como ayuda en su reconocimiento y clasificación. "así como para crear un mejor conocimiento del criterio para juzgar los grados y tipos de toxemia".

Infarto $A_{\text {. }} \rightarrow$ Se reconoce por su color blanco y consistencia firme. En la colección Ciba, en el volumen de Sistema Reproductivo, proponen llamarlo "nódulo" para permitir diferenciarlo de los verdaderos infartos e impedir su asociación con las lesiones placentarias de las toxémicas. Su factor etiológico es aparentemente una esclerosis gradual que afectó inicialmente las pequeñas terminaciones de las arterias placentarias en el margen de la placenta o cerca de ella. Las vellosidades circunvecinas llegan a una lenta declinación que en un periodo de uno o varios meses se va extendiendo. Ya que la superficie de la vellosidad sirve como un endotelio a la sangre de los tabiques intervellosos, la lenta 
necrosis induce a trombosis de la sangre materna que gradualmente se hialiniza causando el infarto, cuya consistencia llega a ser firme y de color blanco por ausencia de circulación. Al microscopio, las vellosidades aparecen pálidas, embebidas en una sustancia rosada hialina homogénea. Bartholomew cree que este tipo de infarto previene la difusión de productos tóxicos dentro de la circulación materna, debido a que su formación se hace muy lentamente ya que la hialinización intervellosa y la trombosis previene la difusión de éstos.

Infarto $B$. - De color amarillo y de consistencia firme. Se encuentra más cerca del margen de la placenta. Su factor etiológico es aparentemente una esclerosis o endarteritis obliterante de características más vivas que en el infarto A. El color amarillo se debe a que en las trombosis de los canales sanguíneos intervellosos que están hialinizados completamente, las células rojas degeneradas no han desaparecido del todo. Inicialmente puede haber un edema ligero transitorio, o presentarse albuminuria $o$ un aumento discreto en la presión sanguínea diastólica, pero pronto se hace incapaz de causar toxemia por las razones anteriormente mencionadas.

Infarto C.-Se reconoce por su color amarillo pardo y su consistencia moderadamente firme. Usualmente se encuentra en el margen de la placenta o cerca de él, pero puede ocurrir en cualquier parte. Como factor etiológico, Bartholomew dice que, es posiblemente una oclusión arterial progresiva. La hialinización de la sangre intervellosa trombosada es incompleta y hay una circulación activa intervellosa en algunas partes del infarto. Su color amarillo pardo es debido a la degeneración parcial de las células rojas embebidas en redes de fibrina. Bartholomew incrimina a este infarto de que permite la difusión de pequeñas cantidades de guanidina de histamina en la circulación materna dando pequeñas manifestaciones de toxemia. Además dice que puede ser responsable del edema moderado, albuminuria e hipertensión.

Infarto D.-Es agudo y puede encontrarse tanto en los períodos iniciales como en los finales de la toxemia. De color café, cuya consistencia es mucho más firme que el tejido normal que lo rodea. Puede presentarse en la superficie materna o dentro ce la placenta. Como factor etiológico parece que se trata de oclusión o trombosis de una pequeña arteria placentaria. Bartholomew atribuye al traumatismo de los movimientos fetales la 


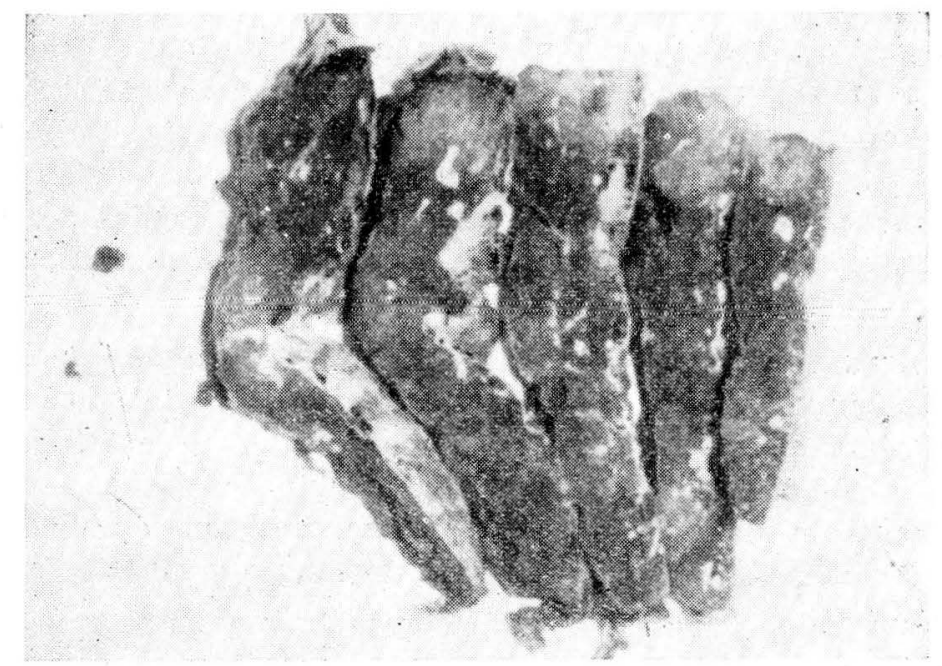

PLACENTA.-Cortes después de fijación de 10 a 12 días. Contrastan las zonas infartadas con el tejido normal.

causa posible de las rupturas del endotelio. La necrosis de las vellosidades se hace mucho más rápidamente que en el infarto C. Microscópicamente las vellosidades se ven pobremente teñidas, muestran mayor degeneración y algunas desintegración.

Bartholomew dice, que, debido a la mayor concentración de peptona la trombosis está inhibida y por lo tanto la histamina y la guanidina tienen un acceso más fácil a la circulación materna produciendo una mayor evidencia de toxemia aguda. El color del infarto es café debido a la degeneración de las células rojas y su consistencia no es tan firme como en los infartos A, B, C, debido a un menor grado de trombosis y a la ausencia de hialinización. Algunas de las vellosidades muestran marcada distensión y ruptura de los capilares terminales. Para Bartholomew el hallazgo de este tipo de infarto indica que la paciente tiene de un moderado a un severo grado de toxemia, el cual termina en eclampsia y por lo tanto sugiere que debe practicarse la inducción del parto ante la persistencia por varias semanas de aumento de la presión sanguínea y de albúmina. Cree que el tamaño y el número de infartos, llevan una relación definida con la celeridad de la toxemia.

Infarto E.-Es mucho más agudo que los anteriores y se puede encontrar en cualquier parte de la placenta. Se reconoce por su color oscuro negro y de consistencia ligeramente más fir- 
me, lo cual da a la superficie una apariencia brillante y que refleja la luz. Su color negro es debido a detención de la sangre venosa en las vellosidades congestionadas cuyos capilares están distendidos o rotos. Las vellosidades muestran distención de la mayoría de los capilares, algunos de los cuales pueden romperse con estravasación de sangre fetal formando coágulos oscuros.

Los infartos D y E se pueden presentar tanto en los casos de abruptio placentae como de eclampsia. La localización de los infartos es importante en el grado de separación de la placenta, ya que aquéllos que se localizan en el margen dan pequeñas separaciones y el feto puede sobrevivir. En cambio, los que se localizan en el centro de la placenta tienden a separar completamente ésta con la consiguiente estravasación de sangre y muerte fetal.

Resumiendo hasta aquí, podemos decir que las lesiones A, B, C, D y E, son verdaderos infartos con aumento progresivo de los mismos. Mientras la consistencia del infarto sea menos firme y el color más oscuro, será mayor el grado de toxicidad. El tamaño y el número de los infartos tiene una relación directa con la severidad de la toxemia.

Infarto $F$. - Se reconoce como una excavación en la sustancia placentaria. Parece como que las vellosidades coriales fueran empujadas hacia un lado, probablemente por una repentina estravasación de la sangre fetal de una vellosidad rota. Bartholomew muestra la gran frecuencia con que ocurre tanto en las placentas normales como en las patológicas y por lo tanto no se las puede atribuir únicamente a la toxemia. Le atribuye una etiología traumática o mecánica, debido a los movimientos fetales en ios últimos meses.

Infarto G.-Es una colección de sangre rodeada por tejido placentario normal o ligeramente comprimido. Si su origen es reciente, aparece negro o muy oscuro, pero a medida que aumenta la edad, el cambio en la hemoglobina llega a ser ocre con ligeras estriaciones que se vuelven amarillas y finalmente blancas, opacas en color. Teóricamente la etiología es la misma que aquella de la lesión $\mathrm{F}$, pero la sangre estravasada es probablemente incompatible con la sangre materna, de aquí que un coágulo determina una excavación.

Infarto $H$.-Se reconoce como una colección de un material parecido a la jalea, transparente, suave, en la sustancia de la placenta. 


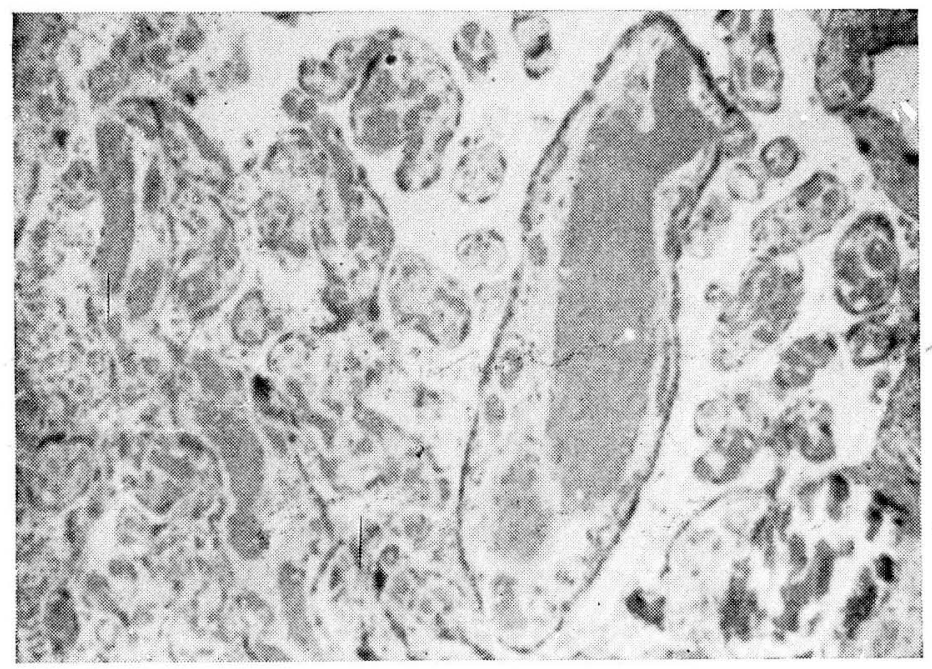

PLACENT'A.-Aspecto microscópico x 10. Vellosidad corial con capilar que la ocupa en casi su totalidad y que presenta depósito de fibrina.

Para resumir: las lesiones F, G y $\mathrm{H}$, son probablemente debidas a extravasación de sangre fetal, de vellosidades capilares terminales que se han roto debido a compresión pasajera de la vena umbilical. Si la sangre materna y fetal son compatibles, la colección de sangre permanece fluída y se drena fácilmente dejando un área excavada (F). Con una mayor incompatibilidad, la lesión puede variar desde un coágulo como jalea transparente (H) a un firme coágulo (G), el cual puede cambiar de color desde el negro hasta el café, el amarillo y finalmente al blanco opaco que es color de fibrina. Ninguna de estas lesiones tiene relación con la toxemia, al menos que esté asociada con infartación aguda, en cuyo caso la hemorragia es oscura.

\section{MATERIAL}

Las lesiones placentarias de toxemias en fresco son oscurecidas por sangre y solamente pueden ser reconocidas cuando han sido previamente fijadas en formol, para identificarlas tanto macroscópica como microscópicamente. Es ésta una de las explicaciones de por qué los hallazgos patológicos no son tan constantes en todos los casos investigados, ya que la identificación de los infartos es difícil si no se ha fijado la placenta con formol durante un tiempo mínimo de una semana, siendo más aconsejado de 3 a 4 semanas. 
Se colectaron unas 200 placentas de casos de pre-eclampsia, eclampsia, hipertensión y abruptio. "De éstas, seleccionamos 56 casos y 10 de abruptio placentae, los cuales reunian todas las condiciones de diagnóstico y exámenes de laboratorio, y deshechamos las otras por no llenar estas normas, o por ser casos de ciagnóstico difícil. También se eliminaron algunas en las cuales el tejido placentario no estuvo bien conservado o debido a una historia incompleta.

Tomamos en todos los casos 5 muestras por lo menos, distribuídas así:

1) Porción proximal del cordón; 2) Cordón distal; 3) Borde placentario en la inserción de las membranas y seno marginal; 4) Cotiledón; 5) Espacio intercotiloidiano.

En algunos otros casos seccionamos áreas con infartos y examinamos también amnios y corión.

Se fijaron por espacio de 6-16 días en solución de formol al $10 \%$. Se practicaron cortes y se colocaron con hemotoxilinaeosina.

En la actualidad se están practicando coloraciones con weigert/verheeff para la identificación del tejido elástico, con una modificación de la coloración periódica ácida de Schiff (P. A. S.) para localizar los muco-polisacárides y con la técnica de Van Gieson para el tejido conectivo y el músculo.

Burstein y colaboradores han hecho microincineraciones para el estudio en campo oscuro de la distribución mineral.

Con las muestras escogidas seguimos para su estudio el mismo criterio utilizado por Bartholomew, es decir, examinar la placa, analizarla, practicar el diagnóstico y luego hacer una correlación entre los hallazgos microscópicos y el caso clínico. De esta manera Bartholomew logra el diagnóstico de toxemia y la severidad de ésta, a partir de una placenta desconocida en la mayoría de los casos.

Para contar el número y darnos cuenta del tamaño de las vellosidades, seguimos la técnica propuesta por Burstein y colaboradores. Un vidrio con áreas cuadriculadas se acopla al ocular del microscopio y se cuentan los haces sincitiales y vellosidades en un promedio de 10 campos.

\section{PATOLOGIA}

De las placentas estudiadas seleccionamos para este capitulo 56, dejando de lado las que no reunían todas las condiciones ya mencionadas. Por otra parte estudiamos 10 placentas más, que se reportan en el capítulo dedicado al abruptio placentae. 


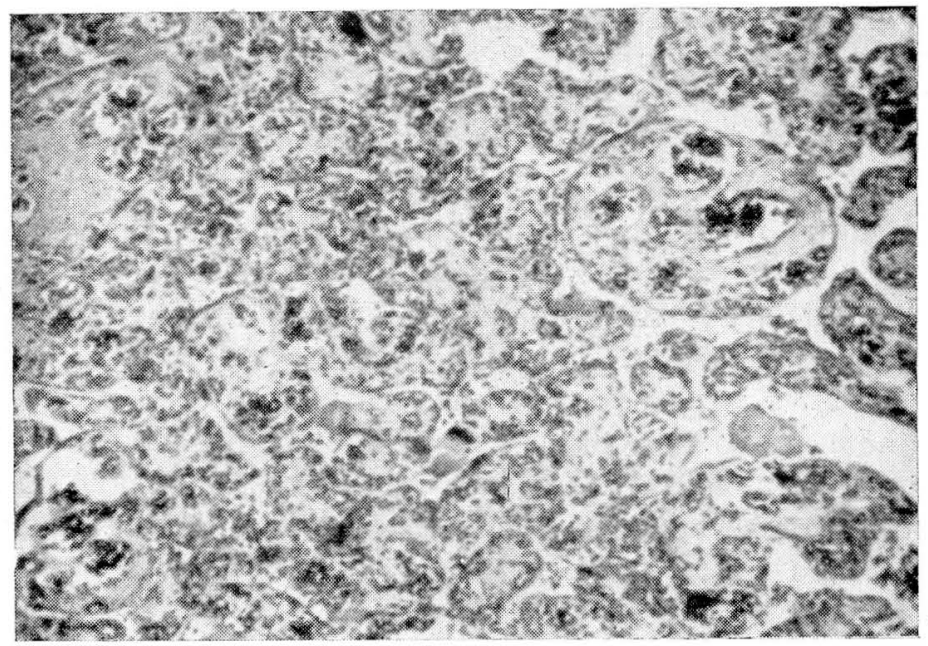

PLACENTA.-Aspecto microscópico $\mathrm{x}$ 10. Infarto D. Vellosidades ensanchadas por dilatación capilar. Dentro de la luz del capilar, glóbulos rojos abundantes. Disminución del espacio intervelloso. Degeneración sincitial.

Queremos hacer un breve resumen de las lesiones microscópicas encontradas en las placentas de pacientes toxémicas. La sucesión en que colocamos las lesiones se basa no sólo en la importancia de las mismas sino en el orden lógico en que se van efectuando las lesiones.

1ạ Dilatación de los capilares vellosos con presencia de 20 o más células rojas dentro de su luz. Esta dilatación conduce a que eì capilar, a veces, llegue a ocupar toda la vellosidad.

2a Ensanchamiento de la vellosidad, la que por su tamaño llega a ponerse en contacto con las vecinas que a su vez se encuentran sufriendo el mismo proceso, determinando así la disminución o desaparición del espacio intervelloso.

$3^{\text {a }}$ Signos de necrosis en los núcleos de las células de las vellosidades manifestado por picnosis, cariorrexis y cariolisis.

4a Circulación intervellosa reducida.

5. Ruptura de los capilares de las vellosidades, debido a sobredistensión por la dificultad del retorno de la sangre venosa de la vellosidad.

6a Aumento de los depósitos y trombos de fibrina.

Los cambios anteriores parecen deberse a congestión. Hay otros cambios determinados $r$ r isquemia de la ve $e_{2}^{-}$sidad, a saber: 
19 Vellosidades pobremente teñidas, con fenómenos de degeneración y desintegración.

20 Desaparición del sincitio.

3o Aparición de acúmulos de núcleos sincitiales.

40 Aumento de las zonas de calcificación.

50 Aumento del número de vellosidades por campo. También aumento de los haces sincitiales.

6 Dilatación y exanguinidad de los capilares fetales (no se encuentran glóbulos rojos dentro de su luz).

70 Lesiones vasculares (esclerosis arterial).

De acuerdo con los datos consignados en las historias clínicas, hemos agrupado las 55 placentas de nuestro estudio en cuatro grupos: pre-eclampsia leve, pre-eclampsia severa, eclampsia y enfermedad cardio-vascular hipertensiva con toxemia sobreagregada.

Pre-eclampsia leve.-Estudiamos 23 placentas, de las cuales pudimos hacer diagnóstico de los diferentes tipos de infarto de la clasificación de Bartholomew, en 7 de ellas que nos resultaron àistribuídas así:

Con infarto tipo C: 4 casos (M-56-38; M-57-1364; M-57-1406; M-57-1467).

Con infarto tipo D: 4 casos (M-57-1366; M-57-1368; M-571459 y M-57-1363).

Pre-eclampsia severa.-Estudiamos 20 placentas, cuyos diferentes tipos de infartos pudimos agrupar asi:

Con infarto tipo C: un caso (M-57-1295).

Con infarto tipo D: 10 casos (A-56-1; M-56-28; M-56-151; M-56-128; M-57-1414; M-57-1418;

M-57-1422; M-57-1445; M-57-

1465; M-57-2110).

Con infarto tipo E: 2 casos (M-57-1460 y M-57-1409).

Es decir, 13 casos de infartos sobre 20 placentas estudiadas.

Eclampsia.-Encontramos 7 infartos sobre un total de 10 placentas estudiadas. Su repartición por grupos fue la siguiente:

Con infarto tipo $\mathrm{C}$ : un caso (M-57-1390).

Con infarto tipo D: 3 casos (A-55-46; A-55-103 y M-57_1447).

Con infarto tipo $\mathrm{H}$ : 3 casos (M-56-3028; M-57-2031 y M-572104).

H. C. V. D. con toxemia sobre-agregada.-Estudiamos solamente tres placentas, de entre las cuales, solo pudimos hacer 


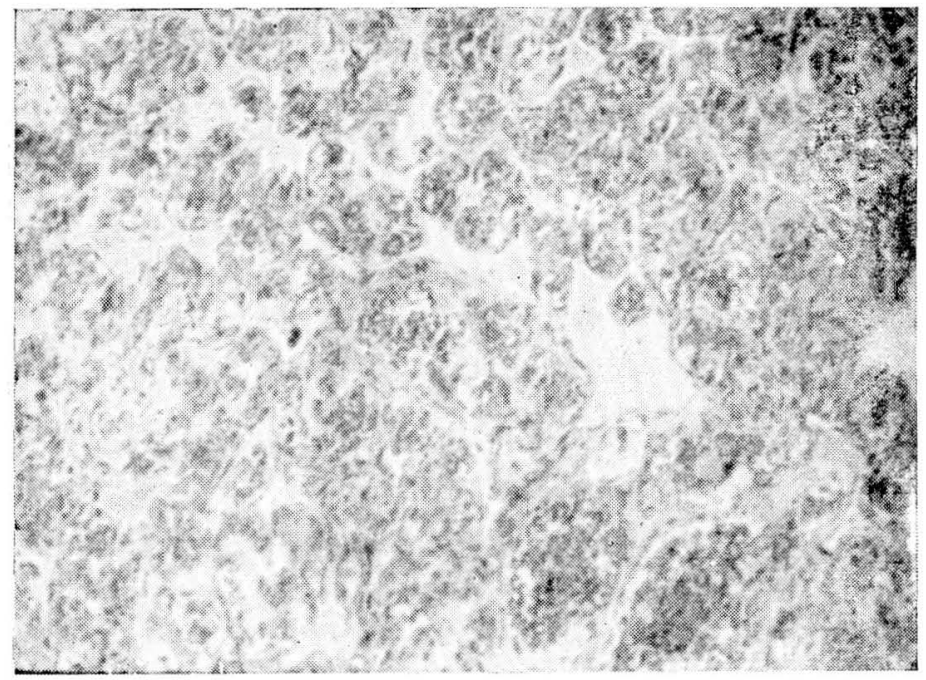

PLACENTA.-Aspecto microscópico x 10. Infarto E. Distensión de todas las vellosidades por aumento de los capilares. Pérdida de la circulación intervellosa. Ruptura de algunos capilares con extravasación capilar.

diagnóstico de infarto en un caso. Es un infarto del tipo D y corresponde al protocolo número M-57-152.

Debemos hacer la aclaración de que en el resto de las placentas estudiadas en cualquiera de los cuatro grupos, no pudimos hacer, tal vez por falta de práctica en la recolección de los especímenes, clasificación alguna de infarto, si bien encontramos numerosos y variados elementos patológicos en todas.

Cordón umbilical.-En los casos de fetos muertos fue frecuente el hallazgo de coágulos dentro de los vasos umbilicales (M-57-1295 preeclampsia leve) y de áreas hemorrágicas (M-571364 y M-57-1406, ambos casos también de preeclampsia leve).

De los resultados anteriores podemos deducir que nuestros hallazgos están ajustados a las experiencias publicadas por Bartholomew, puesto que la presencia de los tipos de infarto y el número encontrado de cada uno de ellos estuvo en proporción directa con la gravedad de los hallazgos clínicos.

\section{COMENTARIO}

La clasificación propuesta por Bartholomew nos parece que identifica las lesiones placentarias de las toxemias y las diferencia en gran manera del resto de hallazgos patológicos presentes en las demás placentas. 
Creemos que si se siguen con todo cuidado las diferentes etapas de la técnica propuesta por Bartholomew, en especial lo referente a la buena fijación del material y a la toma de las muestras en los sitios en donde el aspecto macroscópico lo haga más aconsejable, los resultados de los estudios serán más concluyentes.

Así, en un alto porcentaje de casos, es factible hacer el diagnóstico del tipo de toxemia tomando como base únicamente los hallazgos patológicos en la placenta desconocida.

Los hallazgos patológicos del infarto $\mathrm{C}$, se caracterizan por vellosidades pobremente teñidas con fenómenos de degeneración y desintegración. Es un infarto isquémico por oclusión arterial progresiva.

El infarto D se produce por oclusión o trombosis de pequeñas arterias placentarias y se caracteriza por marcada distención de la mayoría de las vellosidades y ruptura de los capilares terminales.

Las lesiones del infarto $\mathrm{D}$, son producidas por detención de la sangre venosa de los capilares de la vellosidad y se caracteriza por un ensanchamiento de la mayoría de los capilares, ruptura đe algunos con extravasación y formación de coágulos perifocales. En ocasiones los capilares están tan distendidos que llegan a ocupar toda la vellosidad, y se marca en tal forma el ensanchamiento de la vellosidad que al reunirse con las vecinas que sufren el mismo proceso, hacen desaparecer los espacios intervellosos. Hay además pignosis, cariorrexis y cariolisis de los núcleos sincitiales.

Hemos encontrado una estrecha relación entre la lesión patológica y la severidad de la toxemia. Así en la pre-eclampsia leve hay una mayor incidencia del infarto tipo $C$, pero se encuentran algunas lesiones de infarto tipo D. En la pre-eclampsia severa se encuentra una predominancia del infarto tipo D y algunos casos de infarto tipo $\mathrm{H}$. En la eclampsia predominan los tipos D y E sobre los demás. En los casos de abruptio placentae, se encontró una predominancia del infarto tipo $\mathrm{H}$.

Los infartos de tipo C, D y E, son característicos de la toxemia y su número está de acuerdo con la severidad.

Creemos que en un futuro, al mejorar las técnicas de recolección y conservación de material y con una mayor práctica en el diagnóstico y clasificación de los diferentes tipos de lesiones, podramos llegar a un mayor porcentaje de diagnósticos exactos que el que hemos podido tener hasta ahora. 
PATOLOGIA PLACENTARIA

\section{PREECLAMPSIA LEVE}

\begin{tabular}{|c|c|c|}
\hline No. de Placa & Diagnóstico Clínico & Hallazgos Patológicos \\
\hline M_56_38 & Pre_eclampsia leve. & $\begin{array}{l}\text { Infartos recientes. Esclerosis vascu- } \\
\text { lar. Zonas de calcificación. Infartos C. }\end{array}$ \\
\hline M_56-1137 & 32 semanas. TA. $150 / 90$ & $\begin{array}{l}\text { Edema de las vellosidades. Grasa en } \\
\text { el estroma. }\end{array}$ \\
\hline M_57_1334 & $\begin{array}{l}40 \text { S. TA: } 145 / 80 . \text { Ed. } \\
++ \text {. Alk. }++ \text {. }\end{array}$ & $\begin{array}{l}\text { Vellosidades coriales en período re_ } \\
\text { gresión. Placenta envejecida. }\end{array}$ \\
\hline$M-57-1340$ & $\begin{array}{l}40 \text { S. TA: } 160 / 100 . \text { Ed. } \\
\text { ( }-) \text { Alb. }+.\end{array}$ & Ningún hallazgo patológico. \\
\hline M_57_1341 & 40 semanas. & $\begin{array}{l}\text { Zonas de hialinización y fibrosis en } \\
\text { las vellosidades coriales; disminución } \\
\text { del espacio intervelloso. }\end{array}$ \\
\hline M_57-1363 & $\begin{array}{l}40 \text { S. TA: } 130 / 80 . \text { Ed. } \\
+++ \text { Alb. }+\end{array}$ & $\begin{array}{l}\text { Placenta a término con pequeñas } \\
\text { áreas de calcificación. Areas de hemo- } \\
\text { rragia en el cordón umbilical. Infarto } \\
\text { D. }\end{array}$ \\
\hline
\end{tabular}

M_57_1364 40 semanas.

Areas hemorrágicas en cordón umbi_ lical. Conglomerados de núcleos alrededcr de las vellosidades y pequeñas calcificaciones. Infarto $\mathbf{C}$.

M_57-1365 40 semanas. No hay lesiones especiales. Placenta
a término. Normal .

\begin{tabular}{|c|c|c|}
\hline M-57_1366 & $\begin{array}{l}40 \text { S. TA: } 140 / 90 . \text { Alb. } \\
++. \text { Ed. }+.\end{array}$ & $\begin{array}{l}\text { Vellosidades envejecidas con conglo_ } \\
\text { merados nucleares del trofoblasto y } \\
\text { depósitos de fibrina. Areas calcifica. } \\
\text { das. Infarto D. }\end{array}$ \\
\hline M-57_.1368 & $\begin{array}{l}30 \text { semanas. Feto }+. \\
\text { TA: } 130 / 100 . \text { Alb. }++. \\
\text { Ed. }++.\end{array}$ & $\begin{array}{l}\text { Vellosidades envejecidas con áreas } \\
\text { de calcificación. }\end{array}$ \\
\hline N__57_1369 & 40 semanas. & $\begin{array}{l}\text { Conglomerados de núcleos en las ve- } \\
\text { l1!osidades y depósitos de fibrina que } \\
\text { tienden a unirlas. Normal. }\end{array}$ \\
\hline$M-57 \_1375$ & $\begin{array}{l}\text { Gemelar- } 40 \text { S. TA: } \\
\text { 135/95. Alk. }+++. \text { Ed. } \\
+++.\end{array}$ & $\begin{array}{l}\text { Conglomerados núcleares del trofo- } \\
\text { blasto, depósitos de fibrina. Normal. }\end{array}$ \\
\hline M_57_1406 & $\begin{array}{l}\text { 34. S. TA: } 140 / 80 . \text { Ed. } \\
+ \text { Alb. +. Desp. pla- } \\
\text { centario. eFto +. }\end{array}$ & $\begin{array}{l}\text { Areas hemorrágicas del cordón um- } \\
\text { bilical (feto muerto) envejecida de ve_ } \\
\text { llosidades, conglomerados celulares y } \\
\text { depósitos de fibrina. Infarto } \mathrm{C} \text {. }\end{array}$ \\
\hline
\end{tabular}




\begin{tabular}{cccc}
\hline No. de Placa & Diagnóstico Clínico & Hallazgos Patológicos \\
\hline M-57-1408 & $\begin{array}{l}40 \text { S. TA: 135/75. Ed. } \\
++ \text { Alb. }\end{array}$ & $\begin{array}{l}\text { Areas hemorrágicas en cordón. Con- } \\
\text { glomerado de los núcleos del trofo- } \\
\text { blasco y depósitos de fibrina en vello- } \\
\text { sidades; zonas de infarto reciente. }\end{array}$ \\
\hline
\end{tabular}

\begin{tabular}{lll}
\hline M_57_1424 & $\begin{array}{l}\text { 40 S. TA: 140/90. Ed. } \\
++ \text { Alb. }(-) .\end{array}$ & $\begin{array}{l}\text { Conglomerados nucleares en las ve- } \\
\text { llosidades y depósitos de fibrina. }\end{array}$ \\
\hline M-57-1425 & $\begin{array}{l}\text { 40 S. TA: 140/90. Alb. }+ \\
\text { Ed. }+.\end{array}$ & $\begin{array}{l}\text { Conglomerados nucleares y depósi. } \\
\text { tos de fibrina en las vellosidades. }\end{array}$ \\
\hline M_57_1426 & $\begin{array}{l}\text { 40 S. TA: 140/90. Alb. } \\
\text { +. Ed. }+.\end{array}$ & $\begin{array}{l}\text { Vellosidades congestivas. Depósitos } \\
\text { de fibrina abundante. }\end{array}$ \\
\hline M_57_1443 & $\begin{array}{l}\text { Embarazo gemelar. } \\
37 \text { semanas }\end{array}$ & $\begin{array}{l}\text { Conglomerados celulares y depósitos } \\
\text { de fibrina en algunas vellosidades. Ca- } \\
\text { pilar que ccupa toda la vellosidad y } \\
\text { lleno de fibrina. }\end{array}$ \\
\hline
\end{tabular}

\begin{tabular}{lll}
\hline M-57_1452 & 40 semanas. & $\begin{array}{c}\text { Conglomerados nucleares en las ve- } \\
\text { llosidades. }\end{array}$ \\
\hline M_57-1459 & $\begin{array}{l}40 \text { semanas. TA: } 130 / 80 \\
\text { Ed. }+ \text {. Alb. }+.\end{array}$ & Ve!los con grupos nucleares y fibri- \\
& na. Infarto D.
\end{tabular}

\begin{tabular}{lll}
\hline M-57_1467 $\begin{array}{l}\text { Gemelar a término. } \\
\text { TA: 140/90. Alb. +. Ed. } \begin{array}{l}\text { Vellosidades pequeñas, trofoblasto } \\
++.\end{array} \\
\text { to C. }\end{array}$ \\
\hline M_57_2003 Pre-eclampsia leve. & $\begin{array}{l}\text {,Hematoma retroplacentario. Vellos. } \\
\text { con conglomerados nucleares y depó- } \\
\text { sitos de fibrina. }\end{array}$
\end{tabular}

M_57_3106 Pre_eclampsia leve.

Vellosidades pequeñas vascularizadas. Cúmulos de fibrina. Hemorragias.

\section{PRE_ECLAMPSIA SEVERA}

M-55-1634 Embarazo a término. Infartos placentarios blancos. Escle_ rosis marcada de los vasos arteriales.

\begin{tabular}{lll}
\hline A_56_1 & $\begin{array}{l}\text { 30 semana. TA: } 170 / 90 \\
\text { Ed. }+ \text {. Alb. }++++.\end{array}$ & $\begin{array}{l}\text { Características de placenta normal } \\
\text { a término. Infarto D. }\end{array}$ \\
\hline M_56-28 & $\begin{array}{l}40 \mathrm{~S} . \text { TA: } 150 / 100 \mathrm{Ed} . \\
++.\end{array}$ & $\begin{array}{c}\text { Infartcs isquémicos recientes. Infar } \\
\text { to C. }\end{array}$ \\
\hline M_56-128 & Pre_eclampsia severa? & $\begin{array}{c}\text { Vellosidades envejecidas. Arterioes- } \\
\text { clerosis poco marcada. Infarto D. }\end{array}$ \\
\hline M_56_151 & $\begin{array}{l}40 \text { S. TA: } 210 / 120 . \text { Alb. } \\
+++. \text { Ed. }++.\end{array}$ & $\begin{array}{c}\text { Infartos recientes. Necrosis en la de- } \\
\text { ciciua inflamación aguda. Infarto D. }\end{array}$ \\
\hline
\end{tabular}




\begin{tabular}{|c|c|}
\hline No. de Placa & Hallazgos Patológicos \\
\hline M-56_153 & $\begin{array}{l}\text { TA: } 140 / 80 . \text { Ed. }++++ \text { Estructura placentaria normal. En } \\
\text { Alb. }++.40 \mathrm{~S} \text {. Desp. el cordón los vasos muy dilatados. } \\
\text { parcial placentario. }\end{array}$ \\
\hline M_57-498 & $\begin{array}{l}36 \text { S. TA: } 140 / 90 \text { Ed. Infartos, necrosis y calcificaciones. } \\
++ \text { Alb. }++++. \\
\text { Infarto. D. }\end{array}$ \\
\hline M_57_1295 & $\begin{array}{l}\text { Despr. plac. } 30 \text { sem. TA: Coágulos dentro de la vena umbili- } \\
\text { 130/7C. Ed. +. Alb.. cal. Areas necróticas maternas pla- } \\
++++ \text {. Feto +. } \\
\end{array}$ \\
\hline M-57_1348 & $\begin{array}{l}40 \text { S. TA: } 140 / 100 \text { Alb. } \begin{array}{l}\text { Conglomerados nucleares en células } \\
+++. \text { Ed. }+++.\end{array} \text { del trofoblasto y depósitos de fibrina. }\end{array}$ \\
\hline M_57_1349 & $\begin{array}{l}38 \text { S. Gemelar. TA: } 160 \text { Gran congestión de vasos sanguíneos } \\
/ 100 \text { Alb. }+. \text { Ed. }+. \quad \begin{array}{l}\text { vellosidades. Núcleos de trofoblastos } \\
\text { conglomerados. Areas de calcificación. }\end{array}\end{array}$ \\
\hline$M-57-1367$ & $\begin{array}{l}\text { Envejecimiento prematuro de las ve- } \\
\text { llosidades. Conglomerados nucleares y } \\
\text { depósitos de fibrina. }\end{array}$ \\
\hline
\end{tabular}

$\begin{array}{ll}\text { M_57_1407 } & \begin{array}{l}40 \text { S. TA: } 175 / 95 . \text { Alb. } \\ (-) \text { Ed. }+++.\end{array} \\ & \begin{array}{l}\text { Vellosidades con capilares congestio- } \\ \text { nados y dilatados. }\end{array}\end{array}$

M_57_1409

Areas hemorrágicas en las vellosida_

30 S. TA: 220/100 Alb. des. Infarto reciente. Tipo E.

+ .

\begin{tabular}{lll}
\hline M-57-1414 Parto gemelar. Prema- & Acúmulo de células del trofoblasto. \\
turos. 36 S. TA: 150/ Zonas de hialinización en vellosida- & des. Disminución del espacio interve- \\
100. Alb. +++ Ed. +++ & $\begin{array}{l}\text { dloso. Infarto D. Depósito de fibrina } \\
\text { subcoriónica. }\end{array}$
\end{tabular}
M_57-1418 Gemelar 36 S. TA: Envejecimiento de las vellosidades, 150/100 Alb. +. Ed. ++ atrofia de las mismas y zonas de hia_ linización. Infarto D. Zonas de ensan. chamiento con dilatación capilar con zonas de contraste normal.

\begin{tabular}{lll}
\hline M-57_1422 & $\begin{array}{l}40 \text { S. TA: 160/100 ++. } \\
\text { Alb. }+.\end{array}$ & $\begin{array}{l}\text { Vellosidades congestivas. Infarto D. } \\
\text { Necrosis de las vellosidades; ruptura } \\
\text { capilar. }\end{array}$ \\
\hline M_57_1445 & $\begin{array}{l}37 \text { S. TA: 180/120. Ed. } \\
++. \text { Alb. }+.\end{array}$ & $\begin{array}{l}\text { Vellosidades ligeramente congestio- } \\
\text { nadas con conglomerades nucleares. } \\
\text { Infarto D. }\end{array}$
\end{tabular}




\begin{tabular}{ccl}
\hline No. de Placa & Diagnóstico Clínico & \multicolumn{1}{c}{ Hallazgos Patológicos } \\
\hline M_57_1460 & 40 S. TA: 180ü110. Alb. & Conglomerados nucleares en vellosida- \\
& +++ Ed..+++ & des y abundantes depósitos de fibrina. \\
& & Infarto E. Conglomerados de los nú- \\
& & cleos del sincitio. Dilatación capilar \\
& que ocupa la vellosidad en varios si \\
& tios. Pérdida del espacio intervelloso.
\end{tabular}

\begin{tabular}{ccc}
\hline M_57-1465 & $\begin{array}{l}40 \text { S. TA: } 160 / 100 \text { Alb. } \\
+++ \text { Ed. }++.\end{array}$ & $\begin{array}{l}\text { Vellos pequeños con trofoblasto po- } \\
\text { co activo. Vasos engrosados. Depósito } \\
\text { de fibrina en vía de organización. In- } \\
\text { farto D. }\end{array}$ \\
\hline M-57-2110 & Pre_eclampsia severa & $\begin{array}{l}\text { Cordón normal. Extensas zonas de } \\
\text { infarto consistentes en depósitos de fi- } \\
\text { brina organizada y hialinización de al- } \\
\text { gunas vellosidades. Infarto D. }\end{array}$
\end{tabular}

\section{ECLAMPSIA}

A_55_46 Eclampsia. Embd 30 s. Presencia de bacilos en proximidaTA: 180/100. Feto + . des de decidua. Lo demás, normal.

No hay dato de Alb.

ni Ed. gangrena gaseo-

sa.

\begin{tabular}{|c|c|}
\hline A_55_103 & $\begin{array}{l}37 \text { S. TA: } 180 / 120 . \text { Ed. } \\
+++ \text { Alb. }+ \text {. Feto }+ \text {. Necrcsis ce la decidua. Infarto D. } \mathrm{D} . \\
\text { Hemorragia subcoriónica. }\end{array}$ \\
\hline M-56-3028 & $\begin{array}{l}38 \text { S. TA: } 200 / 100 \text { Ed. Calcificaciones. Degeneración sinci_ } \\
++++ \text { Alb. }+.\end{array}$ \\
\hline M-56_3043 & $\begin{array}{l}40 \text { S. TA: } 130 / 90 \text { Ed. Areas de hialinización. } \\
++.\end{array}$ \\
\hline M_57_1390 & $\begin{array}{ll}30 \mathrm{~S} . \text { TA: } 160 / 90 \mathrm{Ed} .+ & \text { Envejecimiento de los vellos. Edema, } \\
\text { Alb. }++++. & \begin{array}{l}\text { congestión y pequeñas áreas hemorrá- } \\
\text { gicas. Infartos C. }\end{array}\end{array}$ \\
\hline M_57_1444 & $\begin{array}{l}40 \text { S. TA: } 150 / 110 \mathrm{Ed} \text { Conglomerados nucleares y depósi_ } \\
+ \text { Alb. }++++.\end{array}$ \\
\hline M_57-1447 & $\begin{array}{l}34 \text { S. TA: } 180 / 90 \text { Alb. Conglomerados nucleares y depósitos } \\
++++. \text { Ed. }++.\end{array}$ \\
\hline M-57_2031 & $\begin{array}{l}\text { Desp. prematuro. } 31 \text { S. } \text { Vellosidades pequeñas. Trofoblasto } \\
\text { TA: } 180 / 100 . \text { Alb. }+++ \text { envejecido vasos engrosados. Infarto } \\
\text { Ed. }++.\end{array}$ \\
\hline M-57_2039 & $\begin{array}{ll}40 \text { S. TA: } 210 / 100 \text { Alb. } & \text { Vellos pequeños. Aún se encuen- } \\
+++. \text { Ed. }++. & \text { tran células de Hofbauer y los ca- } \\
& \text { pilares se encuentran muy dilatados. }\end{array}$ \\
\hline M_57_2104 & $\begin{array}{l}\text { Marcada congestión de las vellosi- } \\
\text { dades. Infarto } \mathrm{E} \text {. }\end{array}$ \\
\hline
\end{tabular}




\section{ENFERMEDAD CRONICA HIPERTENSIVA}

\begin{tabular}{llll}
\hline No. de Placa & \multicolumn{2}{c}{ Diagnóstico Clínico } & \multicolumn{2}{c}{ Hallazgos Patológicos } \\
\hline M-57-152 & $\begin{array}{l}38 \text { S. TA: 220/130 Alb. } \\
++++ \text { Ed. }++.\end{array}$ & $\begin{array}{c}\text { Vasos umbilicales dilatados. Trom- } \\
\text { bos fibrina. Infarto D. }\end{array}$
\end{tabular}

M_57_202 E. C. H. con Tox. No hay hallazgos patológicos. DeSobreag. 30. S. TA: pósitos de fibrina. Infartos A y B. 280/150. Ed. + . Alb.

+++ . Feto + .

M_57_1058 23 S. TA: 260/100 Ed. Envejecimiento prematuro. Engrosa_ + Alb. +. Hemiplejía. miento de la pared de los vasos sanPerd. de viri desde ha- guíneos. Infartos recientes. Cordón ce 1 año. edematoso. 


\section{HOJA DE PLACENTA}

Nombre:
Edad:
Fecha:
Historia $\mathrm{N}^{\mathrm{o}}$
Patología No

Paridad: Gravidez: Fecha parto: Edad embarazo: sems.

HISTORIA OBSTETRICA:

PARTO (Espont.) (Inducido). Ruptura de membranas: (Espont.) (Artif.) hace: hrs.

Duración total del trabajo:

NIÑO: Peso: grms. (Término) (Prematuro) (Vivo) (Muerto) (Macerado) . COMPLICACIONES:

MEDICACION:

PLACENTA: Peso: grms. Dimensiones (Diámetros mayores) cms.

CORDON: Longitud: cms. Inserción: (Central) (Paracentral) (Marginal)

(Velamentosa) (Várices) (Nudos) (Gelatinoso).

MEMBRANAS: (Completas) (Incompletas) (Meconio) Diámetro de ruptura: cms.

Laceración: Inserción: (Marginal) (Otras) :

CARA FETAL: Color:

Normal:

Otros:

Fibrina subcoriónica: $\mathrm{O}$ Poca Moderada Abundante

Difusa: Grumosa:

Vasos sanguíneos:

CARA MATERNA: Superficie: (Completa) (Incompleta) (Desgarrada)

Color: (normal) (oscuro) (claro) (otros) :

Calcificación: O Ligera Moderada Marcada

Hemorragia: $O$ Ligera Moderada Marcada

Localización: Edadi probable:

Fisuración: Ligera Moderada Profunda

SUPERFICIE DE CORTE: Color: Normal: Oscuro: Claro: Otros:

Consistencia

Trombosis intervellosa: No Tamaño: Forma:

Localización:

Degeneración quística del trofoblasto:

Infartos: $\quad N^{0}$ Tamaño: Forma: Localización:

TECNICA DEL CORTE: (Rutina) (Especial) (Histoquímica)

Examinada por: $\longrightarrow$ MD. 


\section{DESCRIPCION MICROSCOPICA}

Número de cortes:

MEMBRANAS: Amnios

Corion

DECIDUA: Pequeña:

Moderada :

Abundante:

Vasos:

Antigua

Fibrosis Leve

Fibrinosis

Leve Moderada Severa

Ateroma:

Necrosis:

Hemorragia (Fecha de establ.) Lig. Mod. Severa Seno Marginal:

Trombosis

Ruptura

TROFOBLASTO: Citología Degeneración fibrinoide Deg. quística. Techo: Tabique: Piso:

Syncitio:

Núcleo:

Citoplasma:

VELLOSIDADES: Madurez

Uniformidad

ESTROMA: Células de Hofbauer Colágeno

Edema :

Fibrosis:

VASOS SANGUINEOS: Endotelio

Presencia de glóbulos rojos nucleados

Trombosis

Endarteritis

INFARTOS:

TROMBOSIS INTERVELLOSA:

DIAGNOSTICO:

Doctor 